\title{
On the Concentration Dependence of Wings of Spectra of Spin Correlation Functions of Diluted Heisenberg Paramagnets
}

\author{
V. E. Zobov ${ }^{a, *}$ and M. M. Kucherov ${ }^{b}$ \\ ${ }^{a}$ Kirensky Institute of Physics, Siberian Branch, Russian Academy of Sciences, \\ Krasnoyarsk, 660036 Russia \\ ${ }^{b}$ Institute of Space and Information Technologies, Siberian Federal University, \\ Krasnoyarsk, 660074 Russia \\ *e-mail: rsa@iph.krasn.ru
}

Received February 26, 2016; in final form, April 11, 2016

\begin{abstract}
Singular points of the autocorrelation function on the imaginary time axis that is averaged over the location of spins in the magnetically dilute spin lattice with isotropic spin-spin interaction at a high temperature have been studied. For the autocorrelation function in the approximation of the self-consistent fluctuating local field, nonlinear integral equations have been proposed which reflect the separation of the inhomogeneous spin systems into close spins and other spins. The coordinates of the nearest singular points have been determined in terms of the radius of convergence of the expansion in powers of time, the coefficients of which have been calculated from recurrence equations. It has been shown that the coordinates of singular points and, consequently, the wings of the autocorrelation function spectrum at strong magnetic dilution are determined by the modulation of the local field by the nearest pairs of spins leading to its logarithmic concentration dependence.
\end{abstract}

DOI: $10.1134 / \mathrm{S} 0021364016110138$

The wings of spectra of correlation functions play an important role in the description of slow processes of the establishment of equilibrium in inhomogeneous spin systems. The problems of ergodicity, thermalization, spin transport, and multiparticle localization are currently being solved [1-4]. The shape of a wing is exponential if the spin autocorrelation function has singular points on the imaginary time axis [5-7]. The coordinate of the singular point, which determines this exponent, was calculated for regular spin lattices in [6-9]. In this work, as in [5-10], systems with isotropic spin-spin interaction are considered, although the same approach can also be applied to more complicated systems with anisotropic interaction [9, 1113]. To the best of our knowledge, the variation of the coordinate of the singular point of the autocorrelation function at magnetic dilution has not yet been considered and is studied in this work. We note that the authors of [10] replaced the inhomogeneous system by the regular lattice of spins at the average distance. However, the validity of such replacement is doubtful, since the calculation of the central part of the spectrum of the autocorrelation function [1, 14-18] showed the importance of allowance for the inhomogeneity of the spatial distribution of spins during the determination of the spectrum shape.
We consider a paramagnet with the isotropic spinspin interaction

$$
H=\sum_{i \neq j} b_{i j}\left[I_{i}^{z} I_{j}^{z}+I_{i}^{x} I_{j}^{x}+I_{i}^{y} I_{j}^{y}\right],
$$

where $I_{i}^{\alpha}$ is the $\alpha$ component $(\alpha=x, y, z)$ of the vector spin operator at the $i$ th site, $I=1 / 2$, and $b_{i j}$ is the spinspin coupling constant. In the local magnetic field produced by the spins of the environment, the timedependent contribution is on the same order of magnitude as the static contribution. Anderson and Weiss [19] proposed to describe this fluctuating field by a Gaussian random process in the presence of a large number of neighbors of each spin. Blume and Hubbard [5] proposed a simple equation for the self-consistent inclusion of this field

$$
F_{B}(t)=\exp \left\{-M_{2} \int_{0}^{t} \int_{0}^{t^{\prime}} F_{B}\left(t^{\prime \prime}\right) d t^{\prime} d t^{\prime \prime}\right\},
$$

for the autocorrelation function

$$
F(t)=\operatorname{Tr}\left\{\exp (i H t) I_{i}^{\alpha} \exp (-i H t) I_{i}^{\alpha}\right\} / \operatorname{Tr}\left\{\left(I_{i}^{\alpha}\right)^{2}\right\} .
$$


The autocorrelation function can be expanded in a series in powers of time

$$
F(t)=\sum_{n=0}^{\infty}(-1)^{n} M_{2 n} t^{2 n} /(2 n) !,
$$

the coefficient $M_{2 n}$ of which is the $2 n$th moment of the spectral density of the autocorrelation function.

The Gaussian local magnetic field of the general form causes the rotations of spins around different axes. In the strict consideration, it is necessary to take into account the time sequence order of rotations, which leads to the necessity of corrections to Eq. (2), in which only the first term of the cumulant expansion is retained. Résibois and De Leener [20] proposed a self-consistent nonlinear integral equation for the autocorrelation function with the kernel presented in the form of a series. Retaining the first term, we have

$$
\frac{d}{d t} F_{R}(t)=-M_{2} \int_{0}^{t} F_{R}^{2}\left(t-t_{1}\right) F_{R}\left(t_{1}\right) d t_{1} .
$$

The common property of self-consistent nonlinear equations for the autocorrelation function is the existence of singular points of solutions on the imaginary time axis, near which

$$
F(t) \approx \frac{A}{\left(i t \pm \tau_{0}\right)^{2}} .
$$

The analysis in $[6,7]$ showed that the functions $F_{B}(t)$ and $F_{R}(t)$ on the imaginary time axis serve as the majorant functions for the strict solution $F(\mathrm{t})$ in the Gaussian self-consistent field; therefore, the coordinates of the singular points satisfy the inequalities

$$
\tau_{R}>\tau_{0}>\tau_{B}
$$

where

$$
\begin{gathered}
\tau_{B}=2.221 / \sqrt{M_{2}}, \quad \tau_{R}=2.781 / \sqrt{M_{2}}, \\
\tau_{0}=2.485 / \sqrt{M_{2}} .
\end{gathered}
$$

We study how the coordinate of the singular point of the autocorrelation function varies at magnetic dilution. We take the initial regular lattice and substitute diamagnetic atoms for magnetic ones at the randomly selected sites. We assume that the occupation of different sites is independent and the probability of the maintenance of the magnetic atom at the site is equal to the average concentration of magnetic atoms $c=$ $N_{M} / N$, where $N_{M}$ is the number of magnetic atoms and $N$ is the total number of lattice sites. Dilution changes the magnitudes of moments. As an example, we present the second and fourth moments [21]:

$$
\begin{gathered}
M_{2}=2 c S_{1}, \quad M_{4}=14 c^{2} S_{1}^{2} \\
-4 c^{2} S_{3}+c 4 S_{2}(2-5 c),
\end{gathered}
$$

which are expressed in terms of lattice sums:

$$
S_{1}=\sum_{j} b_{i j}^{2}, \quad S_{2}=\sum_{j} b_{i j}^{4}, \quad S_{3}=\sum_{k \neq j} b_{j k}^{2} b_{i k} b_{i j} .
$$

In the general case, the $2 n$th order moment contains the lattice sums with the number of summations from 1 to $n$. The lattice sum containing $m+1$ lattice indices is multiplied by $c^{m}$. Equations (2)-(5) are derived under the condition that the contribution proportional to $M_{2}^{n}$ in $M_{2 n}$ exceeds the other contributions. It is clear that this condition at weak dilution $(c \sim 1)$ can still be fulfilled and the coordinate of the singular point of the autocorrelation function is expressed in Eqs. (8) in terms of the concentration-dependent second moment $M_{2}$ given in Eqs. (9).

At $c \ll 1$, the situation changes. It is easy to see that the ratio of the fourth moment to the square of the second moment increases with a decrease in the concentration, since the contribution to the moments from close pairs of spins that includes the lower power of the small value $c^{m}$ increases at $c \ll 1$. Kittel and Abrahams [15] emphasized that this indicates the narrowing of the central part of the spectrum. Anderson [14] showed that the shape of the spectrum becomes Lorentzian in the limit of strong magnetic dilution. The variation of the central part of the spectrum in the whole concentration range was described by Dzheparov $[1,18]$.

The wings of the spectrum and high-order moments are determined by the autocorrelation function near the singularity given by Eq. (6)

$$
\begin{gathered}
g(\omega) \approx(|\omega| \rightarrow \infty) \approx A|\omega| \exp \left(-\tau_{0}|\omega|\right) ; \\
M_{2 n} \approx 2 A(2 n+1) !\left(\tau_{0}\right)^{-2 n-2} .
\end{gathered}
$$

Under dilution, such a contribution to the $2 n$th moment with a large combinatory factor should be multiplied by $c^{n}$, while the contribution of the lattice sums with the same summation index has the order of magnitude of $\sim c Z b_{2}^{2 n}$, where $b_{2}$ is the constant of the interaction between the nearest neighbors, and $Z$ is the number of the nearest neighbors. This contribution (contribution from close pairs of spins) becomes less than the contribution given in Eqs. (11) because of the large combinatory factor at rather large $n$ values $n>$ $\tau_{0} b_{2} / \sqrt{c}$.

The irregular location of spins, i.e., the presence of close pairs, close triads, etc., changes the coordinate of the singular point of the autocorrelation function on the imaginary time axis. To estimate this change, it is necessary to find the variation of the self-consistent equations at the division of the spin system into close pairs and other spins ("mass") [1, 22]. The known example of the self-consistent approach to systems with a small number of nearest neighbors is the Bethe lattice or Cayley tree approximation [23]. In this approximation, we previously studied the dependence 
of the coordinate of the singular point of the autocorrelation function of regular spin systems on the number of nearest neighbors and the dimensionality of space $[24,25]$.

We use the representation of the occupation numbers $n_{j}$ of the $j$ th site: $n_{j}=1$ and 0 if a spin and a nonmagnetic atom are located at the $j$ th site, respectively $[1,16-18,21]$. For the systems where the interaction decreases with the distance according to a power law, e.g., dipole-dipole [1] or RKKY (Ruderman-KittelKasuya-Yosida) [10] interaction, each spin formally interacts with all others. However, the interaction with $Z$ nearest neighbors is much larger than the interaction with the distant surrounding [26]. The contribution from the large number of distant neighbors is taken into account in the form of the contribution given by Eq. (2) for the Gaussian local field

$$
D_{i}(t)=\exp \left\{-2 \sum_{j \notin Z} n_{j} b_{i j}^{2} \int_{0}^{t} \int_{0}^{t^{\prime}} F_{j}\left(t^{\prime \prime}\right) d t^{\prime} d t^{\prime \prime}\right\} .
$$

At the same time, the action of the nearest neighbors is taken into account in the Bethe lattice approximation under the assumption of the independence of contributions from $Z$ nearest neighbors:

$$
F_{j}(t)=\prod_{f}^{Z}\left[\left(1-n_{f}\right)+n_{f} F_{i f}^{(1)}(t)\right] D_{j}(t),
$$

where $F_{j f}^{(1)}(t)$ is the contribution to the variation of the correlation function of the spin at the $j$ th site from the direction leading to the $f$ th spin on the Bethe lattice. The time variation of this function is described by the equation

$$
\begin{gathered}
\frac{d}{d t} F_{j f}^{(1)}(t)=-2 b_{j f}^{2} \int_{0}^{t} D_{f}\left(t^{\prime}\right) \\
\times \prod_{k \neq j}^{Z-1}\left[\left(1-n_{k}\right)+n_{k} F_{f k}^{(1)}\left(t^{\prime}\right)\right] F_{j f}^{(1)}\left(t-t^{\prime}\right) d t^{\prime} .
\end{gathered}
$$

The differences of Eq. (14) from Eq. (5) were considered in [24]. First of all, Eq. (5) was written for the complete autocorrelation function given by Eq. (13) taking into account all links going out of the $j$ th site, while Eq. (14) was written for the contribution to the autocorrelation function from one link. Therefore, only one of the two autocorrelation functions referring to the operator at the $f$ th site "dressed" by the interaction with its surrounding remained in the kernel of Eq. (14). In this autocorrelation function, we excluded the interaction with the $j$ th spin already included in the explicit form in Eq. (14); as a result, the number of factors in the product decreased from $Z$ to $Z-1$. In Eq. (5), such a change in the autocorrelation function is ignored under the assumption of the large number of neighbors. Finally, the dynamics of spins in different directions on the lattice is considered independently in Eq. (14), while Eq. (5) describes the correlated dynamics.

Concerning averaging of Eqs. (12)-(14) over the random location of spins, we note that the separation of close and distant spins depends on the problem solved. For the calculation of the center of the spectrum, the decay of the correlation function on the real time axis is studied. Here, the scale is the width of the spectrum, which is proportional to the concentration and is on the order of magnitude of the interaction between spins at average distances [1, 13-18]. Contributions to the correlation function from the terms containing pairs at smaller distances ("close" pairs) decay owing to fast time oscillations. Therefore, the number of such places in the initial regular lattice (the number of close spins) increases with dilution and is much larger than the coordination number of this lattice. On the contrary, calculating the wings of the spectrum, we study the increase in the correlation function on the imaginary time axis. In this case, the contribution of the close pairs increases monotonically; therefore, the nearest neighbors of the initial lattice should be taken as close spins.

On the basis of the aforesaid, we take a simple model spin system, where each spin has a small number $Z$ of nearest neighbors with the same coupling constant $b_{2}=b / \sqrt{2}$, and introduce the notation $c \delta^{2}=$ $M_{2}-c Z b^{2}$ for the total contribution of distant neighbors. Averaging Eqs. (12)-(14) over the random location of spins in the independent-site-occupation approximation, we obtain the equations

$$
K(t)=\left\langle F_{j}(t)\right\rangle=[Y(t)]^{Z} D(t),
$$

where

$$
D(t)=\exp \left\{-c \delta^{2} \int_{0}^{t} \int_{0}^{t^{\prime}} K\left(t^{\prime \prime}\right) d t^{\prime} d t^{\prime \prime}\right\},
$$

and the function

$$
Y(t)=\left[1+c\left(\left\langle F_{j f}^{(1)}(t)\right\rangle\right)-1\right]
$$

satisfies the equation

$$
\begin{gathered}
\frac{d}{d t} Y(t)=-b^{2} \\
\times \int_{0}^{t}\left[Y\left(t_{1}\right)\right]^{Z-1} D\left(t_{1}\right)\left[Y\left(t-t_{1}\right)-(1-c)\right] d t_{1} .
\end{gathered}
$$

Substituting the expansions in powers of time in the form of Eq. (4) for the functions in Eqs. (17), we 


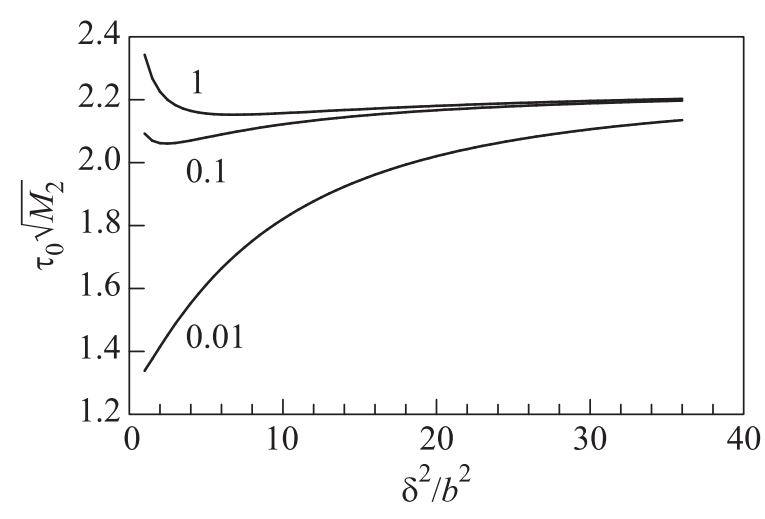

Fig. 1. Coordinate of the singular point versus $\delta^{2} / b^{2}$ at $Z=$ 3 and at three concentration values (numbers near curves).

obtain the following recurrence equations for the coefficients:

$$
\begin{gathered}
Y_{n+1}=b^{2} \sum_{k=0}^{n} G_{n-k} Y_{k}-b^{2}(1-c) G_{n}, \\
G_{n}=\sum_{k=0}^{n}\left(\begin{array}{c}
2 n \\
2 k
\end{array}\right) D_{n-k} Y_{k}^{(Z-1)}, \\
D_{n+1}=c \delta^{2} \sum_{k=0}^{n}\left(\begin{array}{c}
2 n+1 \\
2 k
\end{array}\right) D_{n-k} K_{k},
\end{gathered}
$$

where $Y_{n}, D_{n}$, and $K_{n}$ are the coefficients of terms of order $2 n$ in the expansions of the respective functions in the form of Eq. (4) and $Y_{n}^{(Z-1)}$ is the corresponding coefficient for $[Y(t)]^{Z-1}$. Solving Eqs. (18) numerically, we determine the coordinate of the nearest singular point in terms of the radius of convergence of series (4) for $Y(t)$ calculated by the d'Alembert's formula as the limit of the ratio of the neighboring terms of this series. The calculation results are shown in Figs. 1 and 2.

Figure 1 shows dependences of the coordinate of the singular point $\tau_{0} \sqrt{M_{2}}=\tau_{0} \sqrt{c\left(3 b^{2}+\delta^{2}\right)}$ on $\delta^{2} / b^{2}$ at three concentrations $c=1,0.1$ and 0.01 . At $\delta^{2} / b^{2} \gg 1$, the curves converge to the limit $\tau_{B}=2.221 / \sqrt{M_{2}}$ (8). Figure 2 shows the corresponding concentration dependence $\tau_{0} \sim 1 / \sqrt{c}$ at $\delta^{2} / b^{2}=30$. At lower $\delta^{2}$ values and at strong dilution $c \ll 1$, the concentration dependence of the coordinate of the singular point of the autocorrelation function becomes logarithmic

$$
\tau_{0} \sim-\frac{a}{b} \ln c
$$

which is represented by the line segments in logarithmic coordinates in Fig. 2. Such a dependence is due to the presence of close pairs of spins. Their contribution oscillating rapidly on the real time axis

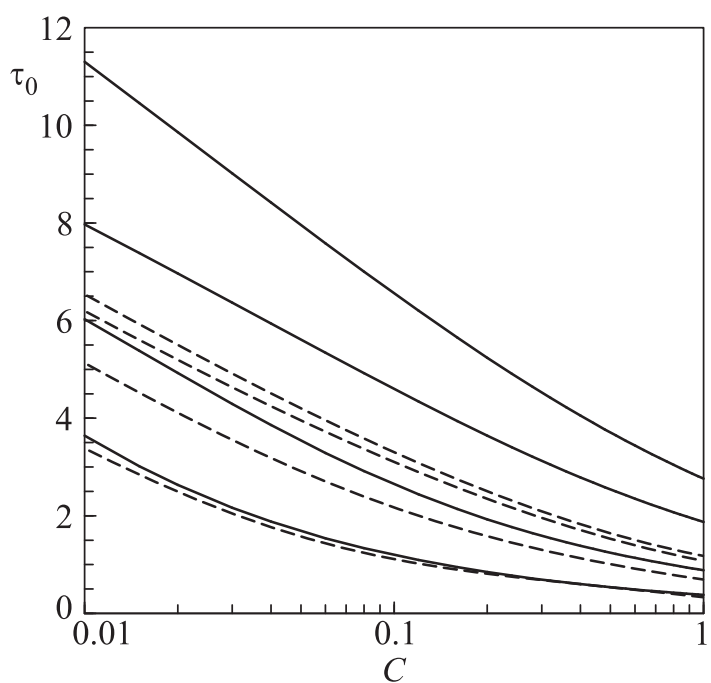

Fig. 2. Concentration dependences of the coordinate of the singular point (in $b^{-1}$ units) at $Z=$ (solid lines) 3 and (dashed lines) 5 at $\delta^{2} / b^{2}=$ (from top to bottom) $0,0.03,3$, and 30 .

$\left\langle F_{j f}^{(1)}(t)\right\rangle \sim \cos b t$ becomes a rapidly increasing contribution $\left\langle F_{j f}^{(1)}(t)\right\rangle \sim \cosh (b \tau) \sim \exp (b \tau) / 2$ on the imaginary time axis $t=i \tau$. However, one pair does not give a singularity. The singular point of the solution of nonlinear equation (17) arises at the combination of signals of many pairs, which occurs under the condition of the fast increase in $Y(t)(16)$, i.e., at $\left.\left(\left\langle F_{j f}^{(1)}(t)\right\rangle c\right)\right\rangle 1$. As a result, the observed dependence of the coordinate of the singular point is obtained in the form of Eq. (19). It can be seen in Fig. 2 that the coefficient $a$ decreases with an increase in $\delta^{2}$ because the function $D(t)$ also contributes to the increase in the kernel of Eq. (17) and reduces the $\tau_{0}$ value. The coefficient $a$ also decreases with an increase in $Z$, since the increase in the exponent of $Y(t)$ results in the increase in the kernel of Eq. (17). On the other hand, such an increase in the exponent leads to the weakening of the dependence on $\delta^{2}$ at $Z=5$ in comparison with that at $Z=2$.

Thus, under magnetic dilution, the central part of the spectrum of the autocorrelation function is transformed from the shape described by the Gaussian curve with exponential wings to the shape described by the Lorentzian curve with exponential wings. The width of the spectrum is a linear function of the concentration $[1,13-18]$, whereas the exponent of the wing is a logarithmic function of the concentration:

$$
g(\omega) \sim A \exp \left\{-\frac{a \omega}{b} \ln \frac{1}{c}\right\}=A c^{\frac{a \omega}{b}} .
$$

Physically, this means that a high frequency arises in the system because of the summation of the frequencies of many pairs $(k \sim \omega / b$ owing to the modulation of the local field. The probability of the formation of $k$ 
pairs is $c^{k}$. At an increase in the modulation frequency, the number of average local frequencies $\omega_{\text {loc }} \sim \sqrt{M_{2}}$ summed in the regular lattice is $k \sim \omega / \omega_{\text {loc }}$. The above analysis provides the following conclusion. It is known that the distances between the majority of spins in magnetically diluted systems are close to the average distance. These spins play an important role in the establishment of equilibrium between parts of the system and determine the center of the spectrum of the autocorrelation function. However, the far wing of the spectrum is determined by clusters of close spins, the probability of the formation of which is small, but the contribution to the modulation frequency is large.

\section{REFERENCES}

1. F. S. Dzheparov, J. Phys.: Conf. Ser. 324, 012004 (2011).

2. J. Eisert, M. Friesdorf, and C. Gogolin, Nature (London), Phys. Sci. 11, 124 (2015).

3. R. Nandkishore and D. A. Huse, Ann. Rev. Condens. Matter Phys. 6, 15 (2015).

4. D. A. Abanin, W. de Roeck, and F. Huveneers, Phys. Rev. Lett. 115, 256803 (2015).

5. M. Blume and J. Hubbard, Phys. Rev. B 1, 3815 (1970).

6. V. E. Zobov, Phys. Lett. A 119, 315 (1986).

7. V. E. Zobov, Sov. J. Theor. Math. Phys. 77, 1299 (1988).

8. V. E. Zobov and M. A. Popov, Theor. Math. Phys. 131, 862 (2002).

9. V. E. Zobov and M. A. Popov, Theor. Math. Phys. 136, 1297 (2003).
10. L. Faoro, L. Ioffe, and A. Kitaev, Phys. Rev. B 86, 134414 (2012).

11. V. A. Atsarkin, G. A. Vasneva, and V. V. Demidov, Sov. Phys. JETP 64, 898 (1986).

12. V. E. Zobov, M. A. Popov, Yu. N. Ivanov, and A. I. Lifshits, J. Exp. Theor. Phys. 88, 157 (1999).

13. H. Hayashi, K. M. Itoh, and L. S. Vlasenko, Phys. Rev. B 78, 153201 (2008).

14. P. W. Anderson, Phys. Rev. 82, 342 (1951).

15. C. Kittel and E. Abrahams, Phys. Rev. 90, 238 (1953).

16. F. S. Dzheparov, A. A. Lundin, and T. N. Khazanovich, Sov. Phys. JETP 65, 314 (1987).

17. E. B. Feldman and S. Lacelle, J. Chem. Phys. 104, 2000 (1996)

18. F. S. Dzheparov, D. V. Lvov, and M. A. Veretennikov, JETP Lett. 98, 484 (2013).

19. P. W. Anderson and P. R. Weiss, Rev. Mod. Phys. 25, 269 (1953).

20. P. Résibois and M. de Leener, Phys. Rev. 152, 305 (1966).

21. J. W. Tuker, J. Phys. C: Solid State Phys. 11, 521 (1978).

22. F. S. Dzheparov and E. K. Khenner, J. Exp. Theor. Phys. 77, 753 (1993).

23. J. Ziman, Models of Disorder: The Theoretical Physics of Homogeneously Disordered Systems (Cambridge Univ., Cambridge, New York, 1979; Mir, Moscow, 1982).

24. V. E. Zobov and M. A. Popov, Theor. Math. Phys. 112, 1182 (1997).

25. V. E. Zobov, Theor. Math. Phys. 123, 511 (2000).

26. A. A. Lundin and B. N. Provotorov, Sov. Phys. JETP 43, 1149 (1976).

Translated by L. Mosina 